\title{
Talaromyces Marneffei Infection in Lung Cancer Patients with Positive AIGAs: A Rare Case Report
}

\author{
Fanhai Lin ${ }^{1,2, *}$ \\ Zhenming Yang ${ }^{1,3, *}$ \\ Ye Qiu ${ }^{4}$ \\ Wen Zeng ${ }^{3}$ \\ Guangnan Liu ${ }^{5}$ \\ Jianquan Zhang'
}

'Department of Respiratory and Critical Medicine, The Eighth Affiliated Hospital, Sun Yat-Sen University, Guangdong, 518000, People's Republic of China; ${ }^{2}$ Department of Pulmonary and Critical Care Medicine, The People's Hospital of Guangxi Zhuang Autonomous Region, Nanning, Guangxi, 5300 I6,People's Republic of China; ${ }^{3}$ Department of Respiratory Medicine, The First Affiliated Hospital of Guangxi Medical University, Nanning, Guangxi, 53002I, People's Republic of China; ${ }^{4}$ Department of Comprehensive Internal Medicine, The Affiliated Tumor Hospital of Guangxi Medical University, Nanning, Guangxi, 53002I, People's Republic of China; ${ }^{5}$ Department of Respiratory Medicine, The Second Affiliated Hospital of Guangxi Medical University, Nanning, Guangxi, 530005,People's Republic of China

*These authors contributed equally to this work

Correspondence: Jianquan Zhang Department of Respiratory and Critical Medicine, The Eighth Affiliated Hospital, Sun Yat-Sen University, No. 3025,

Shennan Middle Road, Shenzhen, 5I8000, Guangdong, People's Republic of China

Tel $+86 \mid 3978$ I 23845

Fax +86755-23482484

Email jqzhang2002@I26.com
Background: Talaromyces marneffei is considered to commonly cause infection in individuals with human immunodeficiency virus (HIV) infection. However, the epidemiology of T. marneffei has changed, and an increasing number of HIV-negative but immunodeficient patients are infected with T. marneffei. The mechanisms of T. marneffei infection of HIV-negative hosts are complex and diverse. We report 2 cases of HIV-negative lung cancer with T. marneffei infection and positive anti-interferon-gamma autoantibodies (AIGAs) to provide clinical experience.

Case Presentation: We report lung adenocarcinoma combined with T. marneffei infection in HIV-negative patients, and their AIGAs were measured. Both patients were male with a family history of cancer and presented with recurrent fever and cough. The patients were negative for HIV antibodies but positive for AIGAs. Chest computed tomography (CT) showed pulmonary nodules, exudative lesions and solid changes. The patients were diagnosed with lung adenocarcinoma and Talaromycosis marneffei (TSM) by pathological examination and tissue culture. Patient 1 received only antifungal treatment, refused antitumor treatment and died in February 2019, and Patient 2 unfortunately died in April 2019 after antifungal and antitumor treatments.

Conclusion: An increasing number of HIV-negative but immunodeficient patients are infected with T. marneffei. The 2 patients in this report had lung cancer and positive AIGAs, causing immunodeficiencies, but the mechanism of T. marneffei infection in such patients is complex. Clinically, we should consider a comprehensive immunological examination to avoid the omission of other immunodeficiencies. We recommend routine testing for AIGA levels in HIVnegative marneffei patients. It is difficult to distinguish between lung cancer and disseminated TSM due to similar clinical characteristics and imaging, and multiple biopsies and cultures of diseased tissue are necessary. Early antifungal treatment and standard antitumor treatment can achieve satisfactory curative effects when a patient has both diseases.

Keywords: Talaromyces marneffei, lung adenocarcinoma, adult immunodeficiency, antiinterferon- $\gamma$ antibodies

\section{Introduction}

Talaromycosis marneffei (TSM) is an opportunistic deep fungal infectious disease caused by Talaromyces marneffei that is found mainly in infected immunodeficient hosts, mainly in Southeast Asia and southern China. In addition to AIDS, these immunodeficiencies also include anti-interferon-gamma autoantibodies (AIGAs), autoimmune diseases, primary immunodeficiency (PID), glucocorticoid and/or immunosuppressive therapy, and malignancy. For HIV-positive hosts, the cellular immune deficiency caused by the decrease in CD4+ T cells is the main mechanism of T. marneffei infection, while the mechanism of HIV-negative host infection with 
T. marneffei is not simple cellular immune deficiency because the total $\mathrm{T}$ cell count, CD4+ T cell count and CD4/ CD8 of the vast majority of these patients are basically normal, including AIGAs and other anticytogenetic diseases, and the inhibition of host immune function does not neutralize peripheral blood $\gamma$-IFN. The mechanism of inhibiting host immune cell function is not well studied. Canceremergent $T$. marneffei infection is mostly diagnosed in the process of treatment, such as chemotherapy and radiotherapy. It is possible that the chemotherapy and radiotherapy cause immune suppression in susceptible hosts, but the T. marneffei infection may be present before treatment; there is little data regarding these possibilities. In addition, tumor factors may lead to host immune dysfunction, and the risk factors are not clear. AIGAs account for the highest proportion (25.1\%) of the risk factors for the susceptibility of HIV-negative hosts to T. marneffei; thus, not all AIGA-positive patients are infected with T. marneffei, suggesting that this immune deficiency is not necessarily the only factor. However, it is not clear how AIGAs inhibit host immune function in cells and through which pathways. Therefore, the mechanisms of T. marneffei infection by HIV-negative hosts are complex and diverse. There may be more than a single immune deficiency when a patient is diagnosed with a malignant tumor. We should consider comprehensive immunological examinations to avoid missing other immunodeficiencies. T. marneffei lung infection and lung cancer have similar imaging features, and they are difficult to differentiate clinically, especially in the same host. Currently, cases of HIV-negative lung adenocarcinoma combined with T. marneffei infection and positive AIGAs are rare. We report 2 cases to improve clinical awareness.

\section{Case Report}

Case 1 was a 63-year-old man who presented with a 4-month history of productive cough and started to deteriorate after having a fever for a week. The patient had an 8-year history of cigarette smoking, and his father had been diagnosed with lung cancer. Repeated antibiotic treatment had been used since he started coughing, which produced a small amount of yellow sputum, and on January 6, 2018, he was diagnosed with communityacquired pneumonia. Unfortunately, his condition did not improve, and he gradually developed persistent right chest pain and shortness of breath after activity. He was admitted to our hospital on March 19th, and chest computed tomography (CT) showed an irregular solid nodule in the anterior segment of the upper lobe of the right lung and lamellar density shadows in the lower lobe of the right lung (Figure 1A and B). A bronchoscopic examination showed that the bronchi were unobstructed, slightly congested and had a mild amount of purulent exudate. A transbronchial lung biopsy (TBLB) was performed in the posterior basal segment and dorsal segment. Galactomannan (GM) of bronchoalveolar lavage fluid (BALF) was 1.068. No fungus, interstitial fibroblastic proliferation, incrassation or fibrosis was seen in the lung tissue biopsy, but acid-fast staining and PAS negativity suggested organizing pneumonia; the patient was then diagnosed with organizing pneumonia. The patient was started on prednisone $30 \mathrm{mg}$ orally once a day on March 27, and he was discharged after his symptoms of cough, chest pain and shortness of breath had gradually improved. He took prednisone as prescribed after

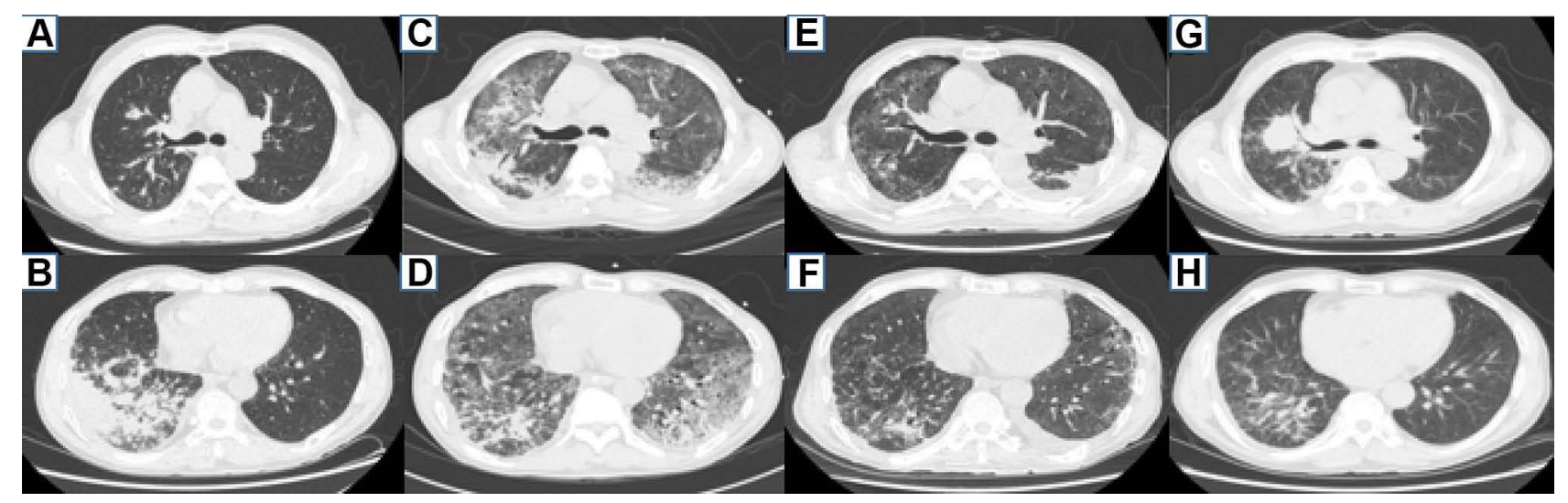

Figure I Patient I chest CT. (A and B) A n irregular solid nodule in the anterior segment of the upper lobe of the right lung and lamellar density shadows in the lower lobe of the right lung on March 20, 2018. (C and D) Multiple patchy high-density shadows are evident in both lungs, with lesions of the right upper lung still being visible during an ICU stay on June 4, 20I8. (E and F) After effective antifungal therapy, the high-density shadow of the two lungs was basically absorbed, the lesion of the right upper lung was almost the same as before, and the left pleural effusion was increased on June 25,2018 . ( $\mathbf{G}$ and $\mathbf{H})$ The nodules in the right upper lobe of the lung were significantly larger, and the lesions in the lower lobes of both lungs were stable on January I5, 2019. 
discharge. However, his condition worsened with a fever that developed on May 8, and he was admitted to the hospital on May 15. On examination, his body temperature was $39.6^{\circ} \mathrm{C}$, and his superficial lymph nodes were not swollen. A small moist rale was detected in the lower right lung. Routine blood tests revealed a leukocyte count of $15.85 \times 10^{9} / \mathrm{L}$, a red blood cell count of $4.18 \times 10^{12} / \mathrm{L}$, a hemoglobin level of $110.70 \mathrm{~g} / \mathrm{L}$, and a neutrophil percentage of $83.0 \%$. His hypersensitive C-reactive protein was $171.23 \mathrm{mg} / \mathrm{L}$, the erythrocyte sedimentation rate was $70 \mathrm{~mm} / \mathrm{h}$, and serum procalcitonin was $0.707 \mathrm{ng} / \mathrm{mL}$. The serum was positive for AIGAs. His plasma HIV antibody, blood culture, beta-D-glucan, GM and Cryptococcus latex agglutination tests were all negative. The lesion in the right upper lobe was still present, and the high-density lesions in the posterior and outer basal segments of the lower lobe of the right lung were slightly smaller on May 16. A percutaneous lung biopsy was performed on May 21, 2018. The right lower lung biopsy showed a chronic inflammatory lesion but no granuloma or neoplastic lesion. Immunohistochemistry with PAS and acid-fast staining showed that the chronic inflammatory lesions were negative. After the admission of the patient and after considering an infection in the lung, the dosage of prednisone was gradually reduced and ultimately discontinued on May 24. The patient was successively treated with imipenem-cilastatin, voriconazole, compounded sulfamethoxazole and linezolid but still had a fever, with the highest temperature being $39.1{ }^{\circ} \mathrm{C}$. On May 30, his symptoms, including difficulty breathing, gradually worsened, and he developed type I respiratory failure. Therefore, he was transferred to the intensive care unit (ICU) for endotracheal intubation for salvage treatment. After admission to the ICU, the patient had scattered herpes in his right anterior chest wall and posterior chest wall. Herpes zoster was clinically diagnosed, and acyclovir sustained release tablets were given as oral antiviral treatment. T. marneffei was cultured from lung biopsy tissue performed on June 4, and chest CT showed growth of the two lung lesions (Figure 1C and D). Liposomal amphotericin B was started on June 6 as an antifungal agent; the patient's temperature returned to normal, and the patient's cough and shortness of breath improved after 3 days. He was transferred back to the general ward. Unfortunately, liposomal amphotericin B was discontinued due to progressive renal impairment and was replaced by voriconazole $0.2 \mathrm{~g}$ twice daily starting on June 19 . He was discharged after a chest CT showed that the lung lesions were significantly smaller (Figure $1 \mathrm{E}$ and F) on June 25. He was started on a course of itraconazole after discharge. He developed another episode of cough and fever with a maximum temperature of $40^{\circ} \mathrm{C}$ on December 25, 2020 . A chest CT showed that the nodules in the right upper lobe of the lung were significantly larger and that the lesions in the lower lobes of both lungs were stable on January 15, 2019 (Figure $1 \mathrm{G}$ and $\mathrm{H}$ ). Bronchoscopy revealed that the mucosa in the anterior segment of the right upper lobe had lost its normal structure, and the lumen was narrow. The pathology from the TBLB performed in the anterior segment of the right upper lobe showed primary adenocarcinoma (Figure 2A-D). The patient was confirmed to have right lung adenocarcinoma and TSM. However, he was discharged after refusing treatment and died in February 2019.

Case 2 was a 57-year-old male from Guangxi Province who presented with cough and fever for a week. He had a 40-year history of smoking, and his father had nasopharyngeal cancer. He was admitted to the hospital with a temperature of $38.5^{\circ} \mathrm{C}$ but with no abnormalities in the other physical examination parameters on January 22, 2018. His white blood cell count was $15.68 \times 10^{9} / \mathrm{L}$, hemoglobin was $91.90 \mathrm{~g} / \mathrm{L}$, and neutrophil percentage was $74.0 \%$. His serum albumin was $27.5 \mathrm{~g} / \mathrm{L}$. His hypersensitive C-reactive protein was $163.71 \mathrm{mg} / \mathrm{L}$. HIV antibody testing was negative. No abnormalities were found in renal function, blood glucose, autoantibody spectrum or T lymphocyte subsets. A chest CT showed multiple nodules in the upper lobe of the left lung, a mass in the left hilar lung and a mass in the middle lobe of the right lung with mediastinal and left hilar lymph node enlargement (Figure 3A and B). Fiber bronchoscopy revealed that the trachea, main bronchus and bronchi segmentales were unobstructed, and no necrotic material or tumor was observed. TBNA of the right paratracheal lymph node and subcarinal lymph node was conducted. The pathology of those lymph nodes revealed purulent exudate and hemorrhage. He was given cefoperazone tazobartan at $2.25 \mathrm{~g}$ every 8 hours and vancomycin at $500 \mathrm{mg}$ every 12 hours a week, but the patient had a recurrent high fever. A chest CT on January 31 showed that the pulmonary lesions had increased in size (Figure 3C and D). T. marneffei was isolated from TBNA tissues on February 4 (Figure 4A and B). Thus, voriconazole at $200 \mathrm{mg}$ orally every 12 hours and amphotericin B at $30 \mathrm{mg} /$ day intravenously were prescribed. However, amphotericin $\mathrm{B}$ was discontinued due to the development 


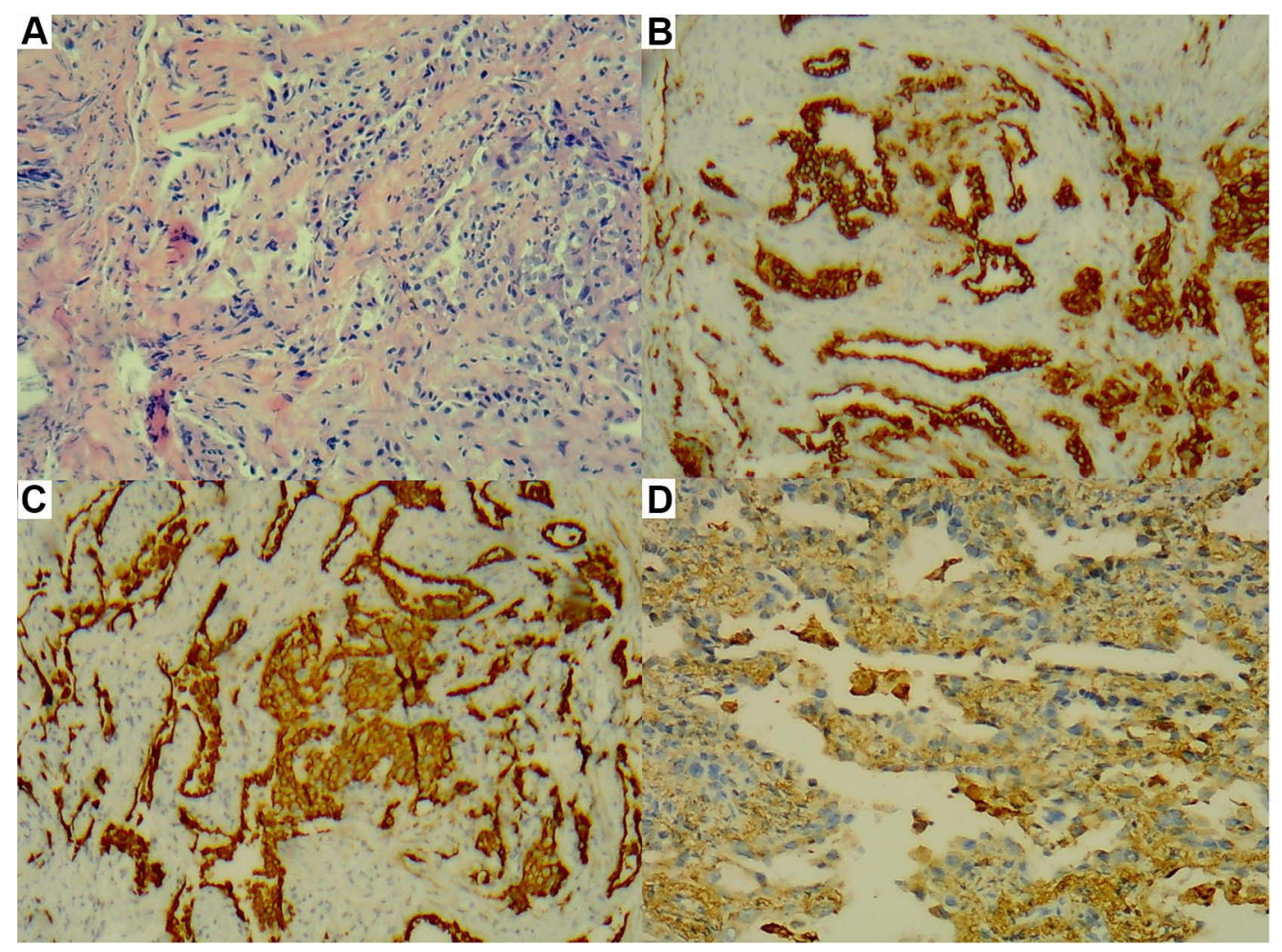

Figure 2 (A-D) There were tumor cells in the upper right lobe, some of which were glandular and micropapillary. (A) HE staining, $\times 200 .($ B) CK (+), $\times 200$. (C) CK7 (+), $\times 200$. (D) Napsin A (+), $\times 200$.

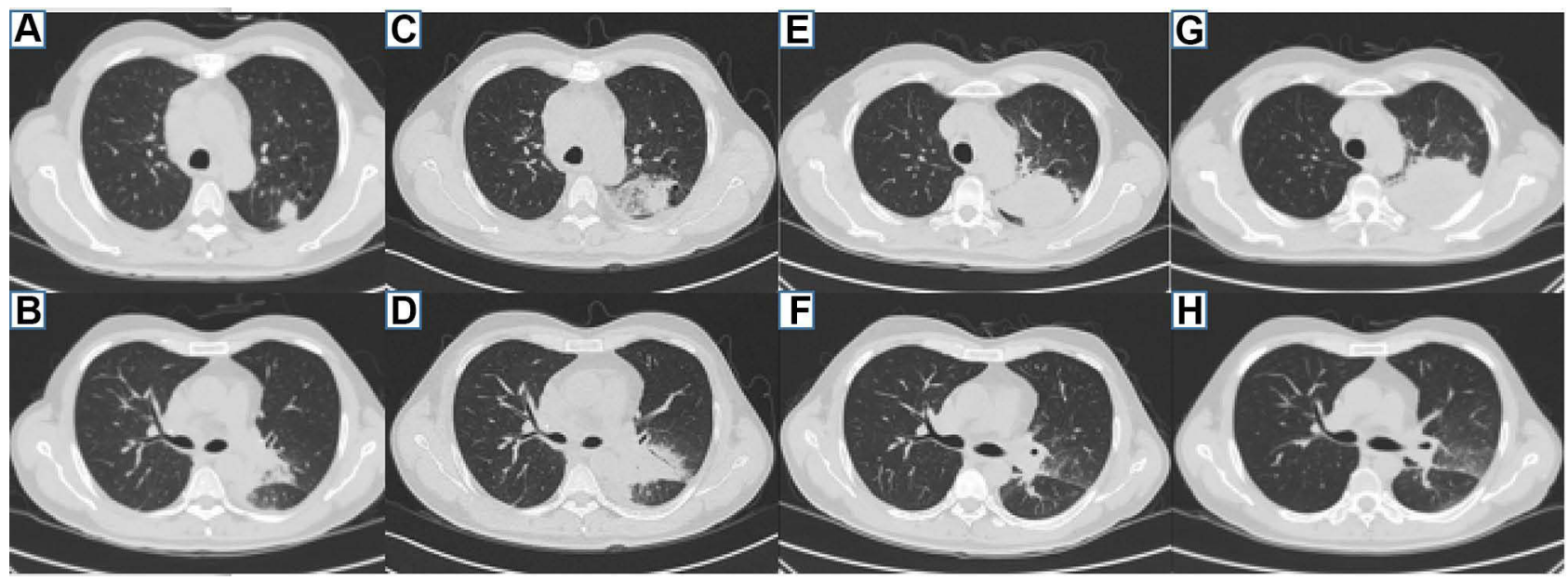

Figure 3 Patient 2 chest CT. (A and B) Multiple nodules in the upper lobe of the left lung and a mass in the left hilar lung on January 24, 2018. (C and D) The lung lesions of the upper lobe of the left lung and left hilar lung increased on January 3I, 20I8. (E and $\mathbf{F}$ ) The lesion in the upper lobe of the left lung was enlarged, and the mass of left hilar was reduced, on August 27, 2018. ( $\mathbf{G}$ and $\mathbf{H}$ ) The lesion in the upper lobe of the left lung continued to enlarge, and the mass of left hilar was the same as before on November 6, 2018.

of renal impairment noted on February 13. The lung lesions were smaller 2 weeks later on February 22, 2018. He was discharged with voriconazole orally $200 \mathrm{mg}$ every 12 hours. However, he repeatedly developed a cough and increased sputum in August 2018, and AIGAs were identified in his serum. There were no intracavitary neoplasms seen during tracheoscopy. TBLB was performed in the posterior segment of the upper lobe of the left lung, and TBNA of the subcarinal lymph node was repeated. TBLB revealed acute and chronic inflammation of the 


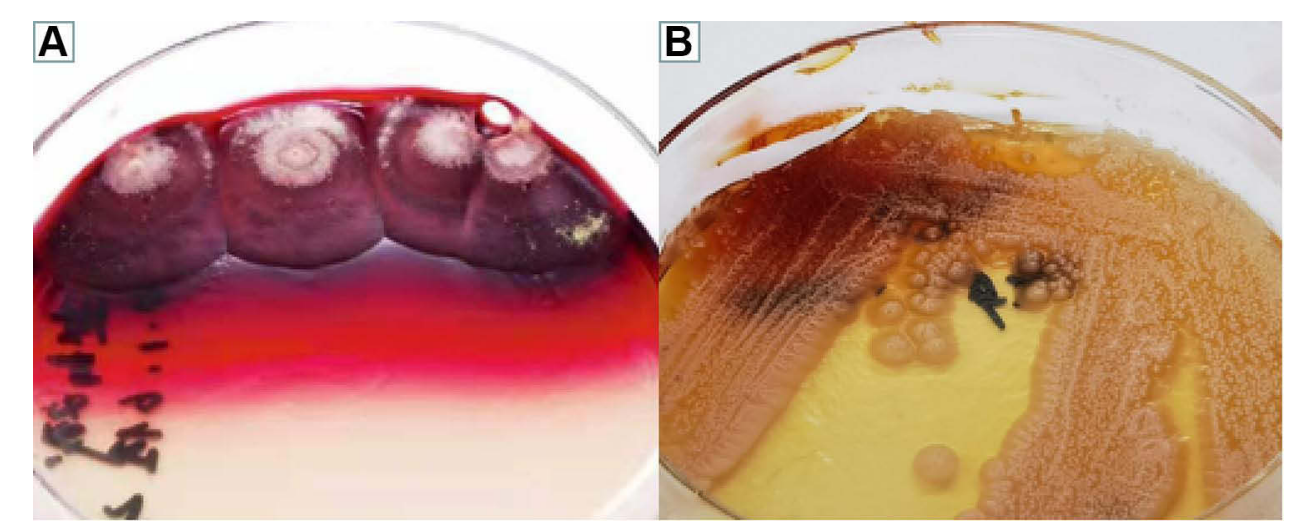

Figure 4 (A and B) Microbiological examination-colony morphology: T. marneffei is thermally dimorphic, growing as a mycelium at $25^{\circ} \mathrm{C}(\mathbf{A})$ and as yeast-like cells at $37^{\circ} \mathrm{C}$ (B) on Sabouraud dextrose agar for two weeks.

mucous membranes accompanied by local hemorrhage, but no cancer cells were observed. No tumor cells were found in the TBNA. We considered that the patient might have T. marneffei combined with a nontuberculosis mycobacteria (NTM) infection. Therefore, rifampin at $450 \mathrm{mg} /$ day, ethambutol at $750 \mathrm{mg} /$ day, moxifloxacin at $400 \mathrm{mg} /$ day, and clarithromycin at $500 \mathrm{mg} /$ day were started. Meanwhile, cefoperazone tazobartan $2.25 \mathrm{~g}$ every 8 hours + linezolamide 0.6 g every 12 hours and voriconazole $200 \mathrm{mg}$ every 12 hours were used for antibacterial treatment and antifungal therapy, respectively. After one week, cefoperazone tazobartan and linezolamide were discontinued, and chest CT on August 27, 2018 showed that the lesion in the upper lobe of the left lung was larger, but the mass of the left hilum was smaller (Figure $3 \mathrm{E}$ and F). The patient was discharged after his temperature gradually recovered. After discharge, he continued anti-NTM and antifungal therapy. However, he presented with left-sided chest pain without fever in October 2018. A chest CT showed that the lesion in the upper lobe of the left lung had enlarged, and on November 6, 2018, the mass of the left hilar was the same as before (Figure $3 \mathrm{G}$ and $\mathrm{H}$ ). An ultrasound-guided lung puncture biopsy was performed on November 12. The pathology was confirmed as infiltrating adenocarcinoma, and no epidermal growth factor receptor mutation was observed (Figure 5A-D). The anti-TNM drugs were discontinued. A positron emission tomography/computed tomography showed lung cancer in the upper lobe of the left lung with metastasis in the lower lobe of the left lung, the left hilum and mediastinal lymph node, the left 3rd and 4th ribs, and the cervical, thoracic and lumbar spine. The final diagnosis was confirmed as primary lung adenocarcinoma (cT4N2M1 stage IV) with metastasis to the lower lobe of the left lung, ribs, thoracic and lumbar vertebrae and a T. marneffei infection. He took itraconazole capsules orally at $100 \mathrm{mg}$ twice a day for antifungal therapy but refused chemotherapy and chose icotinib for antitumor treatment. We did not recommend this treatment because his EGFR status was negative, and he died in April 2019.

\section{Discussion}

Previously, TSM was believed to be common in HIVpositive patients, and its mechanism of infection is mainly due to cellular immune deficiency caused by a decrease in CD4+ T cells. ${ }^{1}$ However, in recent years, it has been found that HIV-negative patients with immunodeficiency in Southeast Asia and Southern China are also susceptible to $\mathrm{TSM}^{2}$ Qiu et $\mathrm{al}^{3}$ found that the common underlying diseases causing T. marneffei infection in HIV-negative patients between 2011-2019 were AIGAs (25.1\%), autoimmune diseases (21.8\%), PID (12.7\%), glucocorticoid and/or immunosuppressive therapy (14.2\%), malignancy (13.1\%), diabetes mellitus $(8.0 \%)$, postorgan transplantation $(7.6 \%)$, etc. Patients with AIGAs had the highest percentage of comorbidities, which causes an associated immunodeficiency called adult-onset immunodeficiency syndrome. Guo et $\mathrm{al}^{4}$ reported 58 HIV-negative patients infected with T. marneffei and the positive rate of AIGAs was $94.8 \%$. None of those patients had a history of cancer, autoimmunity, or any form of immunosuppressive treatment. However, the pathogenesis of TM and AIGAs is not completely clear. $\mathrm{Ku}$ et $\mathrm{al}^{5,6}$ found that the HLADRB1*16:02-DQB1*05:02 haplotype and the HLADRB1*15:02-DQB1*05:01 haplotype were present in Southeast Asian individuals with positive AIGAs. Four alleles were expressed at different rates in individuals, and DRB1*16:02 and DRB1*15:02, DQB1*05:01 and 


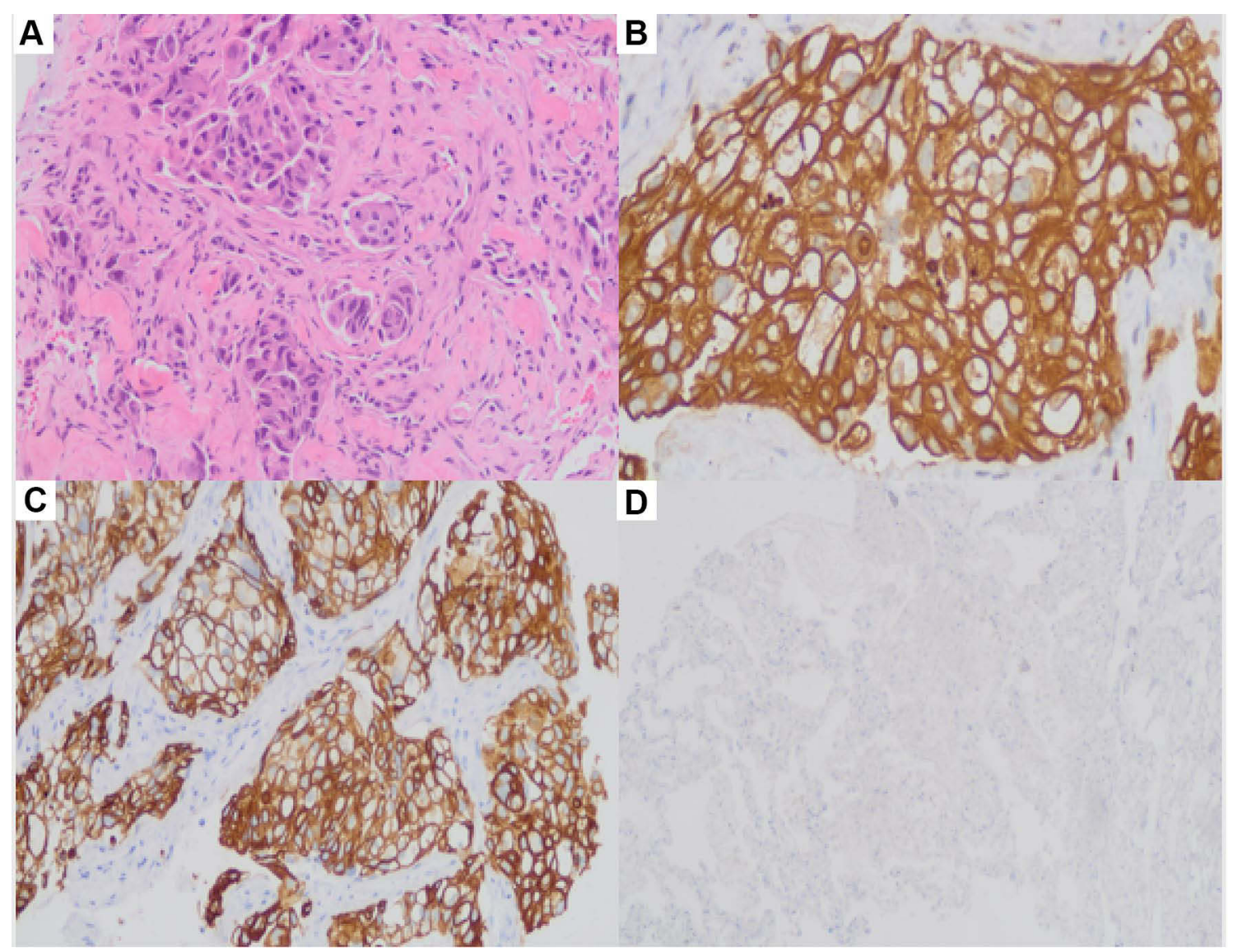

Figure 5 (A-D) Biopsy of the upper lobe of the left lung indicated infiltration of lung adenocarcinoma. (A) HE staining, $\times 200 ;$ (B) CK (+), ×400; (C) CK7 (+), $\times 200 ;($ D) P40 $(+) \times 100$.

DQB1*05:02 had synergistic effects in producing AIGAs. This immunodeficiency mechanism may be one of the reasons why T. marneffei is endemic in Southeast Asia. AIGAs account for the highest proportion (25.1\%) of the risk factors for the susceptibility of HIV-negative hosts to T. marneffei; thus, not all AIGA-positive patients are infected with T. marneffei, suggesting that this immune deficiency is not necessarily the only factor. Canceremergent T. marneffei infection is mostly diagnosed in the process of treatment, such as chemotherapy and radiotherapy. It is possible that the chemotherapy and radiotherapy cause immune suppression in susceptible hosts, but the T. marneffei infection may be present before treatment; there is little data regarding these possibilities. In addition, tumor factors may lead to host immune dysfunction, and the risk factors are not clear. In this report, 2 patients were diagnosed with lung adenocarcinoma; their parents had a history of cancer, and they ultimately were positive for AIGAs. It is worth noting that both patients were already infected with $T$. marneffei prior to antitumor therapy, which indicates that they had two diseases that cause immunodeficiencies: lung cancer and AIGAs. It is necessary to test for the presence of AIGAs in HIVnegative TSM patients with or without cancer, and clinicians should pay more attention to this possibility.

T. marneffei mainly invades the host mononuclear macrophage system, including the skin, lymphatic system, bone marrow and lung, among which, the lung is the most frequently involved organ. Lung cancer and lung T. marneffei infection have similar clinical manifestations but lack specificity. These diseases have symptoms such as high fever, cough, lymph node enlargement, and weight loss. There can be exudate shadows, mass shadows, pleural effusion, pericardial effusion, and mediastinal lymph node enlargement on chest imaging. ${ }^{2,7}$ The gold standard for the diagnosis of talaromycosis is isolating T. marneffei from body samples. At present, the recognized diagnostic standard mainly includes the following. 1) T. marneffei was cultured from clinical specimens (including blood, sputum, bronchoalveolar lavage, etc.) at $25^{\circ} \mathrm{C}$ 
and $37^{\circ} \mathrm{C}$. 2) Yeast-like cells with a central septum were found by D-PAS, PAS or hexamine-silver staining. However, culturing T. marneffei is time consuming because it takes 7-10 days, and the positive rate of T. marneffei in HIV-negative patients is low. It is difficult to distinguish TSM from tuberculosis when the pathology shows chronic granulomatous inflammation. Multiple pulmonary diseases are similar in imaging findings and clinical manifestations but lack specificity. When patients have these diseases at the same time, it is easy to misdiagnose or miss one of them. How to timely and accurately diagnose these diseases and improve the prognosis is critical. Clinically, when a patient is diagnosed with lung cancer or TSM and if the disease progresses further after treatment, the possibility of a misdiagnosis or concomitant disease should be considered, and simultaneous multisite biopsy is recommended to confirm the diagnosis if the patient has systemic multisystem involvement. ${ }^{8}$

TSM treatment in HIV-negative hosts is mainly similar to the TSM treatment regimen of HIV-positive patients because there is a lack of clear treatment guidelines, and these treatments include intravenous amphotericin B $(0.6 \mathrm{mg} / \mathrm{kg} \bullet \mathrm{d})$, which was changed to oral itraconazole $(400 \mathrm{mg} / \mathrm{d})$ for maintenance treatment after two weeks. Voriconazole and posaconazole can also be used in the treatment of TSM. ${ }^{9,10}$ Czaja et $\mathrm{al}^{11}$ reported that using rituximab to inhibit B lymphocytes could reduce the production of AIGAs in plasma. However, Chan et $\mathrm{al}^{12}$ found that rituximab did not significantly reduce the production of AIGAs but might increase the potential risk of T. marneffei infection. Therefore, it is still controversial whether TSM should be treated for AIGAs. In our report, Patient 1 received antifungal treatment and was not willing to be treated for lung cancer, and the patient developed systemic metastasis of lung cancer, which resulted in death. In Patient 2 , his infection was controlled after antifungal treatment, but he was unwilling to accept chemotherapy and chose icotinib for antitumor treatment, although his EGFR status was negative. $\mathrm{Lu}$ et $\mathrm{al}^{13}$ conducted a comparative study of the efficacy of EGFR-TKIs in 84 patients with advanced nonsmall cell lung cancer. The results showed that the DCR of the EGFR mutation-positive group was $95.8 \%$, and the median PFS was 11 months. The DCR of the EGFR mutation-negative group was $0 \%$, and the median PFS was 1 month. DCR and PFS were significantly different between the two groups $(\mathrm{P}<0.05)$. In this case, the patient's treatment choice was unwise because his EGER status was negative. When lung cancer lesions are mixed with
T. marneffei lesions, further examination of the possible underlying diseases is often not performed after obtaining a positive result for a T. marneffei infection, which was the main reason why the lung cancer of these two patients was not diagnosed early. Therefore, extensive lung lesions with T. marneffei should be biopsied at multiple sites to determine if the lesions are of different natures, especially when the patient does not respond to therapy. When lung cancer and TSM coexist, it is important to treat both. Early antifungal treatment and standard antitumor treatment can achieve satisfactory curative effects.

Both lung cancer and positive AIGAs can cause related immune deficiencies, which makes patients susceptible to TM; however, the mechanism remains to be further studied. We can learn the following from the diagnosis and treatments of those 2 patients. 1) The detection of AIGAs in HIV-negative TSM hosts (especially in Southeast Asia) is helpful to evaluate the patients' immune state. This measure should be tested routinely in HIV-negative T. marneffei-infected patients. 2) There is some overlap in some areas between TSM and lung adenocarcinoma, and it is difficult to identify when these two diseases are present at the same time. It is necessary to perform biopsies of multiple sites and sometimes consecutively during different times when there is no change or when there is an increase in size in multiple lung lesions, especially masses or solitary nodules, during antifungal treatment. 3) Early diagnosis is the main prognostic factor. The presence of pulmonary adenocarcinoma in AIGA-positive patients combined with $T$. marneffei infection is rare, and early antifungal treatment and standard antitumor treatment can achieve satisfactory curative effects. 4) Clinically, when a patient is considered to suffer from pulmonary fungal infection, but antifungal treatment is not effective, it is necessary to distinguish tuberculosis, NTM, and opportunistic pneumonia and to exclude the possibility of lung primary lung disease, including tumors.

\section{Abbreviations}

AIGAs, anti-interferon-gamma autoantibodies; PID, primary immunodeficiency; BALF, bronchoalveolar lavage fluid; CT, computed tomography; CTPA, computed tomographic pulmonary angiography; GM, galactomannan; HIV, human immunodeficiency virus; ICU, intensive care unit; NTM, nontuberculosis mycobacteria; PAS, periodic acid-Schiff; TSM, Talaromycosis marneffei; TBLB, transbronchial lung biopsy; DCR, disease control rate; PFS, progression-free survival. 


\section{Data Sharing Statement}

All the data are fully available without restriction.

\section{Ethics Approval and Informed Consent}

This study was approved by the Faculty of Medicine at The First Affiliated Hospital of Guangxi Medical University [2021(KY-E-218)]. All patients or patients' parents provided written informed consent. The study was carried out in accordance with the principles of the Declaration of Helsinki. The first author vouches for the completeness and accuracy of the data and for the fidelity of the study to the protocol.

\section{Consent for Publication}

Signed consent was obtained for the publication of the case details from the participant.

\section{Acknowledgments}

We have received no substantial contributions from nonauthors. Fanhai Lin and Zhenming Yang are co-first authors for this study.

\section{Author Contributions}

FL and ZY contributed to the study concept and design, acquisition of data (imaging features), analysis and interpretation of data, and drafting of the manuscript. YQ, WZ and GL contributed to the analysis and interpretation of the data. JZ contributed to the study concept and design, acquisition of data, analysis and interpretation of data, and revision of the article. All authors contributed to data analysis, drafted or revised the article, gave final approval of the version to be published, agreed on the journal to which the article has been submitted and agreed to be accountable for all aspects of the work.

\section{Funding}

This work was supported by grants from the Natural Science Foundation of China [NSFC81760010 and 82060364] and the Science and Technology Department of Guangxi Zhuang Autonomous Foundation of Guangxi Key Research and Development Program (No. GuikeAB20238025).

\section{Disclosure}

The authors report no conflicts of interest in this work.

\section{References}

1. Jiang J, Meng S, Huang S, et al. Effects of Talaromyces marneffei infection on mortality of HIV/AIDS patients in southern China: a retrospective cohort study. Clin Microbiol Infect. 2019;25 (2):233-241. doi:10.1016/j.cmi.2018.04.018

2. Chan JF, Lau SK, Yuen KY, Woo PC. Talaromyces (Penicillium) marneffei infection in non-HIV-infected patients. Emerg Microbes Infect. 2016;5(3):e19. doi:10.1038/emi.2016.18

3. Qiu Y, Feng X, Zeng W, Zhang H, Zhang J. Immunodeficiency disease spectrum in HIV-negative individuals with Talaromycosis. $J$ Clin Immunol. 2021;41(1):221-223. doi:10.1007/s10875-02000869-5

4. Guo J, Ning XQ, Ding JY, et al. Anti-IFN- $\gamma$ autoantibodies underlie disseminated Talaromyces marneffei infections. J Exp Med. 2020;217 (12):e20190502. doi:10.1084/jem.20190502

5. Ku CL, Lin CH, Chang SW, et al. Anti-IFN- $\gamma$ autoantibodies are strongly associated with HLA-DR*15:02/16:02 and HLA-DQ*05:01/ 05:02 across Southeast Asia. J Allergy Clin Immunol. 2016;137 (3):945-948.e8. doi:10.1016/j.jaci.2015.09.018

6. Pithukpakorn M, Roothumnong E, Angkasekwinai N, et al. HLADRB1 and HLA-DQB1 are associated with adult-onset immunodeficiency with acquired anti-interferon-gamma autoantibodies. PLoS One. 2015;10(5):e0128481. doi:10.1371/journal.pone.0128481

7. Qiu Y, Liao H, Zhang J, Zhong X, Tan C, Lu D. Differences in clinical characteristics and prognosis of Penicilliosis among HIV-negative patients with or without underlying disease in Southern China: a retrospective study. BMC Infect Dis. 2015;15:525. doi:10.1186/s12879-015-1243-y

8. Qiu Y, Lu D, Zhang J, Zhong X, Liu G, Li B. Treatment of disseminated Talaromyces marneffei with tracheal infection: two case reports. Mycopathologia. 2015;180(3-4):245-249. doi:10.1007/ s11046-015-9891-4

9. Lei HL, Li LH, Chen WS, et al. Susceptibility profile of echinocandins, azoles and amphotericin B against yeast phase of Talaromyces marneffei isolated from HIV-infected patients in Guangdong, China. Eur J Clin Microbiol Infect Dis. 2018;37(6):1099-1102. doi:10.1007/ s10096-018-3222-x

10. Sirisanthana T, Supparatpinyo K, Perriens J, Nelson KE. Amphotericin B and itraconazole for treatment of disseminated Penicillium marneffei infection in human immunodeficiency virus-infected patients. Clin Infect Dis. 1998;26(5):1107-1110. doi: $10.1086 / 520280$

11. Czaja CA, Merkel PA, Chan ED, et al. Rituximab as successful adjunct treatment in a patient with disseminated nontuberculous mycobacterial infection due to acquired anti-interferon- $\gamma$ autoantibody. Clin Infect Dis. 2014;58(6):e115-e118. doi:10.1093/cid/ cit809

12. Chan JF, Chan TS, Gill H, et al. Disseminated infections with Talaromyces marneffei in non-AIDS patients given monoclonal antibodies against CD20 and kinase inhibitors. Emerg Infect Dis. 2015;21 (7):1101-1106. doi:10.3201/eid2107.150138

13. Lu T, Li Q, Li L, et al. Results of EGFR mutations detected in pleural effusion and its clinical significance in 132 patients with advanced non-small cell lung cancer: a retrospective study in a single center. Zhongguo Fei Ai Za Zhi. 2020;23(12):1059-1065. doi:10.3779/j. issn.1009-3419.2020.104.23 


\section{Publish your work in this journal}

Infection and Drug Resistance is an international, peer-reviewed openaccess journal that focuses on the optimal treatment of infection (bacterial, fungal and viral) and the development and institution of preventive strategies to minimize the development and spread of resistance. The journal is specifically concerned with the epidemiology of

Submit your manuscript here: https://www.dovepress.com/infection-and-drug-resistance-journa| antibiotic resistance and the mechanisms of resistance development and diffusion in both hospitals and the community. The manuscript management system is completely online and includes a very quick and fair peerreview system, which is all easy to use. Visit http://www.dovepress.com/ testimonials.php to read real quotes from published authors. 